\title{
Identifying targets of the Sox domain protein Dichaete in the Drosophila CNS via targeted expression of dominant negative proteins
}

\author{
Shih Pei Shen ${ }^{1,3}$, Jelena Aleksic ${ }^{1,2}$ and Steven Russell ${ }^{1,2^{*}}$
}

\begin{abstract}
Background: Group B Sox domain transcription factors play important roles in metazoan central nervous system development. They are, however, difficult to study as mutations often have pleiotropic effects and other Sox family members can mask phenotypes due to functional compensation. In Drosophila melanogaster, the Sox gene Dichaete is dynamically expressed in the embryonic CNS, where it is known to have functional roles in neuroblasts and the ventral midline. In this study, we use inducible dominant negative proteins in combination with ChIP, immunohistochemistry and genome-wide expression profiling to further dissect the role of Dichaete in these two tissues.
\end{abstract}

Results: We generated two dominant negative Dichaete constructs, one lacking a DNA binding domain and the other fused to the Engrailed transcriptional repressor domain. We expressed these tissue-specifically in the midline and in neuroblasts using the UAS/GAL4 system, validating their use at the phenotypic level and with known target genes. Using ChIP and immunohistochemistry, we identified two new likely direct Dichaete target genes, commisureless in the midline and asense in the neuroectoderm. We performed genome-wide expression profiling in stage 8-9 embryos, identifying almost a thousand potential tissue-specific Dichaete targets, with half of these genes showing evidence of Dichaete binding in vivo. These include a number of genes with known roles in CNS development, including several components of the Notch, Wnt and EGFR signalling pathways.

Conclusions: As well as identifying commisureless as a target, our data indicate that Dichaete helps establish its expression during early midline development but has less effect on its established later expression, highlighting Dichaete action on tissue specific enhancers. An analysis of the broader range of candidate Dichaete targets indicates that Dichaete plays diverse roles in CNS development, with the 500 or so Dichaete-bound putative targets including a number of transcription factors, signalling pathway components and terminal differentiation genes. In the early neurectoderm we implicate Dichaete in the lateral inhibition pathway and show that Dichaete acts to repress the proneural gene asense. Our analysis also reveals that dominant negatives cause off-target effects, highlighting the need to use other experimental data for validating findings from dominant negative studies.

Keywords: Sox, Dichaete, Drosophila, CNS, Dominant-negative, Genomics

\section{Background}

During the process of development, precise changes in gene expression are critical for correctly timed differentiation and body plan development. Tissue-specific gene expression is often highly conserved across distantly related species and changes in gene expression are

\footnotetext{
* Correspondence: s.russell@gen.cam.ac.uk

'Department of Genetics, University of Cambridge, Cambridge, UK

${ }^{2}$ Cambridge Systems Biology Centre, University of Cambridge, Cambridge, UK

Full list of author information is available at the end of the article
}

thought to contribute to speciation and the evolution of phenotypic diversity [1]. However, the search for direct targets of the transcription factors regulating developmental processes and the identification of regulatory networks remains challenging. In particular, it is difficult to differentiate between downstream effects, functional compensation by related genes and genuine direct targets. In the context of conserved families of transcriptional regulators, research in model organisms utilizing a number of different genetic tools can be helpful in elucidating the precise regulatory mechanisms at play in

\section{Biomed Central}


specific tissues with the hope that such insights are generalisable to a broader range of species.

Group B Sox domain factors represent such a family of conserved transcription factors with roles in a number of important developmental processes, including the regulation of stem cell pluripotency [2], eye development [3], and Drosophila segmentation [4,5] among others. In the context of the central nervous system, early expression of group B Sox genes in the neuroectoderm is detected in all metazoans examined to date, and in at least some cases it is known that this expression is functionally important [68]. However, a degree of functional redundancy between different Sox genes expressed in the same tissues makes this a difficult gene family to study and means that comparatively few direct regulatory targets are known despite the importance of this class of transcription factor [9-11]. In Drosophila there are four group B genes: SoxNeuro $($ SoxN), Dichaete, Sox21a and Sox21b, the last three organised in a genomic cluster that is conserved in insects [1214]. SoxN and Dichaete encode group B1 proteins corresponding to mammalian Sox1 and Sox2, respectively [7]. We have previously shown that the mouse Sox 2 gene is able to effectively rescue Dichaete mutant phenotypes, highlighting the considerable functional conservation exhibited by Sox proteins [15].

Dichaete has an early role in embryonic segmentation, where it acts to regulate the expression of primary pair rule genes, and is then active in the anlage of the central nervous system [4,5]. In the early stages of Drosophila CNS specification, Dichaete and SoxN are required for the correct expression of the proneural gene achaete and in this role they cooperate with the DV-patterning homeodomain proteins Intermediate Neuroblasts Defective (Ind) and Ventral Neuroblasts Defective (Vnd) [16,17]. After gastrulation, Dichaete is dynamically expressed in many segregated neuroblasts and their progeny $[15,18]$. In the developing CNS, Dichaete has a defined phenotype in the ventral midline where it is a uniquely expressed Sox gene early in embryogenesis. It is required for the correct specification and development of midline glial cells, directly regulating the slit gene via interaction with the POU domain protein Ventral Veins Lacking $(\mathrm{Vvl})$ and the PAS domain protein encoded by single minded $[15,19]$. Interestingly, a Sox-POU interaction is also a critical part of early mouse development, where Sox 2 partners with Oct4 $[20,21]$. While the precise role Dichaete plays during the later stages of embryonic CNS development has not yet been clarified, a recent study suggests a role in maintaining the stem cell-like neuroblasts in a self-renewing state, with downregulation of Dichaete leading to exit from the cell cycle and premature differentiation, again showing similarity to the function of Sox2 in mammals [22].

Dichaete and SoxN are both known to be active in the early neuroectoderm and, similarly to mammalian group
B genes, display a degree of functional redundancy or compensation. Mutations in either of these genes exhibit relatively weak phenotypes in cells where they are coexpressed but double mutants show severe neural hypoplasia, suggesting that at least one group B gene is necessary for the correct specification or differentiation of early neural progenitors $[9,10]$. However, while it is clear the fly group B Sox proteins can functionally compensate in regions of the neuroectoderm, a careful analysis of the phenotypes suggests that each protein has a unique role, even in cells that they are co-expressed in. This is most apparent in the intermediate column of the neuroectoderm where Dichaete acts to repress the expression of the proneural gene achaete $(a c)$ whereas SoxN appears to have activating functions $[10,16]$.

To date, the number of characterised direct targets of Dichaete in the developing CNS is comparatively small, including the proneural gene $a c$ in the neuroectoderm and slit in the midline $[10,15,16,19]$. The early embryo genome-wide binding profiles published by the Berkeley Drosophila Transcription Network Project (BDTNP) and the modENCODE consortium identify Dichaete binding at thousands of genomic locations, suggesting a considerable number of direct target genes [23,24]. However, since the binding profiles were obtained using whole embryos, they do not give insight into tissue-specific aspects of Dichaete function. Similarly, due to functional compensation and pleiotropy it is also difficult to tease apart tissue-specific aspects of Dichaete function from the genomic analysis of loss-of-function mutants.

In this study, we probed tissue-specific Dichaete function in vivo, using two newly developed dominant negative Dichaete alleles. We focused on specific neural tissues in the early embryo: the neuroectoderm and the ventral midline. We first characterised the constructs using phenotypic analysis to determine whether their overall effect matches that of Dichaete mutants. We then used a mixture of phenotypic and binding analysis to characterise two new putative Dichaete direct target genes, commisureless (comm) in the midline and asense (ase) in the neuroectoderm. We showed that Dichaete directly binds to regulatory elements associated with these genes. Finally, we expressed the dominant negatives in specific neural tissues and performed whole transcriptome expression profiling, comparing these data with results from existing genome-wide binding studies to identify a set of potential Dichaete targets. Using these data, we also assess the appropriateness of dominant negatives as a tool for unravelling tissue-specific transcription factor function.

\section{Results}

Evaluation of dominant negative constructs

Since Dichaete is widely and dynamically expressed during Drosophila development and null mutations have 
pleiotropic effects $[15,18]$, its function in specific tissues is difficult to study in detail. To facilitate tissue-specific analysis of Dichaete action, we generated dominant negative (DN) Dichaete proteins and expressed them in specific tissues via the GAL4-UAS system [25]. We produced three types of construct: two dominant negative proteins, either lacking the Dichaete HMG DNAbinding domain $(\triangle \mathrm{HMG})$ or a fusion between the full length Dichaete and the strong repressor domain from the Engrailed protein (EnRep), we also produced a full length wild-type Dichaete control. Both types of dominant negative protein have been previously reported in work with vertebrate group B Sox proteins: the EnRep in Xenopus [26], Chick [27] and zebrafish [28], and the $\triangle H M G$ in Xenopus [26]. EnRep fusions are reported to turn transcription factors into strong repressors, effectively shutting down the expression of target genes $[29,30]$. The way that $\triangle \mathrm{HMG}$ proteins act is less clear but it is likely they function by sequestering essential cofactors. Thus our expectations when using the DN constructs was that the EnRep fusion would reveal sets of genes where Dichaete function is required for activation but would not detect genes where Dichaete normally acts as a repressor, whereas the $\triangle \mathrm{HMG}$ construct should uncover genes repressed or activated by Dichaete via interactions with a cofactor. Since we have previously shown that Sox2 effectively rescues Dichaete mutant phenotypes, we also generated a similar set of dominant negative constructs using mouse Sox $2(\mathrm{mSox} 2)$ to test the generality of our approach.

To assess the effectiveness of the DN constructs we expressed each of them in the midline of wild type flies using a sim-GAL4 driver and in neuroblasts using a pros-GAL4 driver. In the case of sim-GAL4 we used a driver line heterozygous for sim-GAL4 on the 2nd and homozygous for sim-GAL4 on the 3rd chromosome crossed to homozygous UAS lines. For pros-GAL4, a 2nd chromosome homozygous driver line was crossed with homozygous UAS lines. In both cases, all progeny express the transgene. We have previously shown that expressing wild type Dichaete or mSox2 with a midline driver rescues Dichaete null mutant phenotypes [15]: both Dichaete $\triangle \mathrm{HMG}$ and EnRep constructs produced Dichaete-like phenotypes in the CNS when expressed with sim-GAL4. BP102 staining of DN-Dichaete expressing embryos showed collapsed commissures, reduced separation between the longitudinal tracts, gaps in longitudinal connectives, misrouting and inappropriate midline crossing by longitudinal axons (Figure 1A-F). Similar phenotypes were observed with the mSox 2 constructs (not shown) and in all cases $50-70 \%$ of embryos ( $n>500)$ exhibited some degree of CNS disruption. In agreement with our previous observations [15], expression of the wild type Sox proteins under the same conditions produced very mild phenotypes (Figure 1A and D). Focusing on the longitudinal connectives as revealed by FasII staining, we observed strong CNS phenotypes with both the fly and mouse DN constructs (Figure 1G-L).

Since slit is a Dichaete target gene in the midline, we examined Slit in DN expressing conditions and found, as expected, a reduction in expression (Figure 1M-P). This observation indicates that, at the level of an individual target gene, the DN constructs mimic Dichaete loss of function. However, with both FasII and BP102 staining, the phenotypes we observed were more severe than those exhibited by Dichaete null mutants. This suggests that the DN constructs affect processes additional to those regulated by Dichaete and to test this we expressed DN constructs in the midline of Dichaete null mutants. Whereas the wild type Sox proteins rescue the CNS phenotypes, expression of DN constructs produced phenotypes more severe than those of Dichaete mutants (Figure 2), supporting the view that the DN constructs, in addition to disrupting normal Dichaete function, are likely to have additional effects and thus act as neomorphic alleles.

Since previous studies indicate that Dichaete acts to repress achaete expression in the neuroectoderm, we used the DN constructs to investigate the effects on achaete expression in the early neuroectoderm using a pros-GAL4 driver [31]. In the stage 11 embryo, $a c$ is expressed in a single medial column neuroblast in each hemisegment [32]. Consistent with previous reports $[16,17]$, in Dichaete mutants we see a variable increase in the number of $a c$ positive cells in the medial column and the appearance of cells in the intermediate column, with up to 5 cells observed in a single hemisegment as well as a more general diffuse staining indicative of low level derepression in the neuroectoderm (Figure 3A and B). Driving the Dichaete or mSox $\triangle \mathrm{HMG}$ constructs with pros-GAL4 results in a similar increase in ac positive cells, with both fly and mouse proteins behaving similarly (Figure 3C and D). In contrast, when constructs carrying the EnRep fusions are expressed under the same conditions we see a loss of $a c$ expression in the medial column and no ectopic expression in the intermediate column (Figure 3E; Table 1). In addition, we found that expressing $\triangle \mathrm{HMG}$ in the early embryo resulted in precocious expression of $a c$, with $50-60 \%$ of Dichaete $^{\triangle H M G}$ or Sox2 ${ }^{\triangle H M G}$ expressing embryos $(n=50)$ showing weak $a c$ expression at stage $6 / 7$ in contrast to wild type expression at stage 8 . These observations support the view that Dichaete directly regulates $a c$ and further confirm that the DN constructs behave as expected. Specifically, we note that expression of the EnRep construct results in a loss of $a c$ expression consistent with its expected activity repressing targets genes. In contrast, the $\Delta \mathrm{HMG}$ construct results in ectopic expression of $a c$ in both medial and intermediate columns, consistent with 


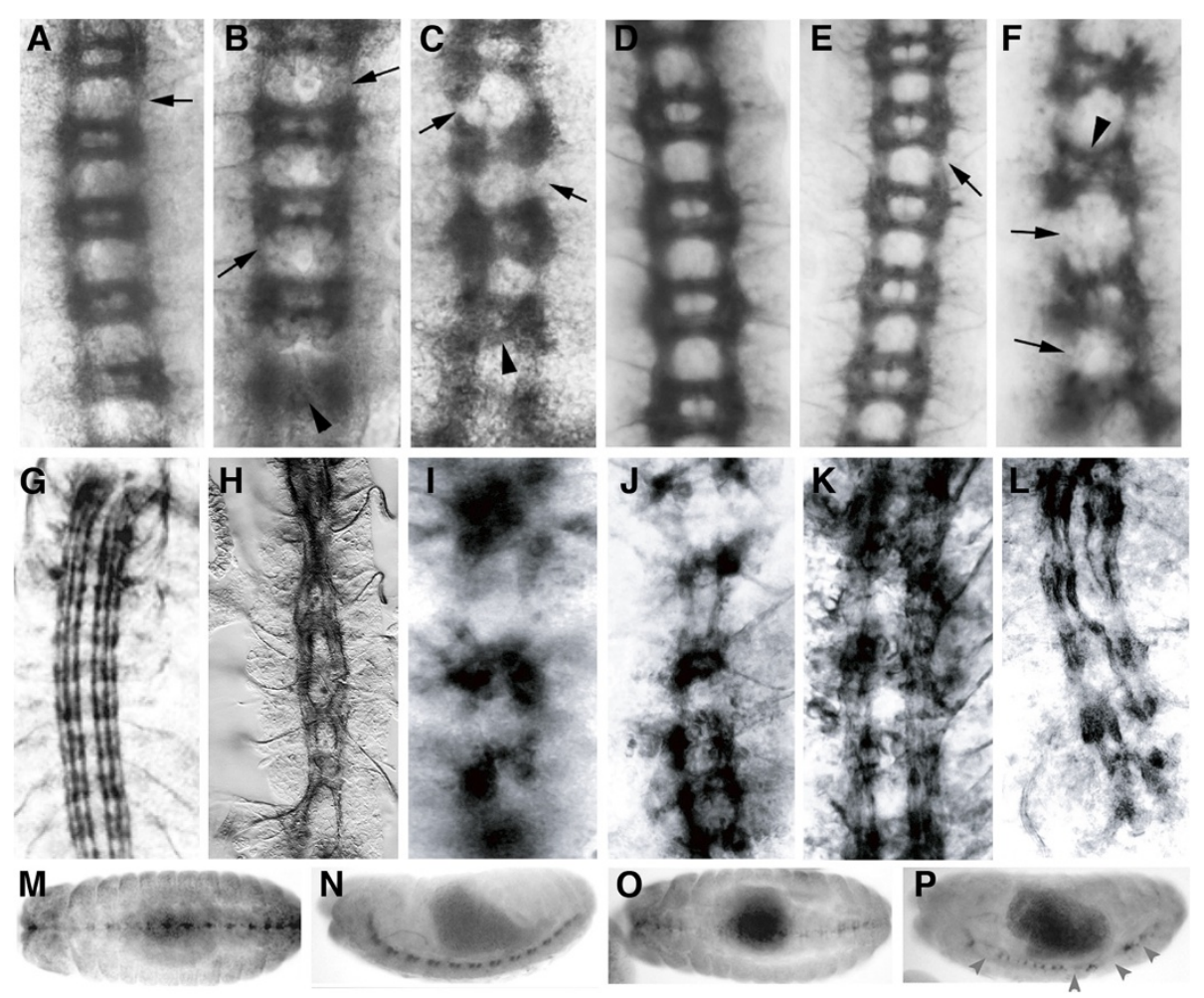

Figure 1 Dominant negative proteins mimic Dichaete phenotypes. A-F) BP102 staining reveals the ventral nerve cord of stage 16 embryos. All ventral views with anterior to the top. A) sim-GAL4;UAS-D; B) sim-GAL4;UAS-D ${ }^{\triangle H M G}$; C) sim-GAL4;UAS-D ${ }^{\text {EnRep; }}$ D) pros-GAL4;UAS-D; E) pros-GAL4; UAS-D $\left.D^{\triangle H M G} ; \mathbf{F}\right)$ pros-GAL4;UAS-D EnRep. Arrows indicate breaks in the longitudinal connectives, arrowheads indicate collapse of commissures. G-L) Fasll staining revealing the major longitudinal fascicles $\mathbf{G}$ ) Wild type; $\left.\mathbf{H}) D^{r 72} / \mathrm{Df}(3 \mathrm{~L}) f z-G S 1 a ; \mathbf{I}\right) \operatorname{sim}-G A L 4 ;$ UAS-D $\left.\left.{ }^{\Delta H M G} ; \mathbf{J}\right) \operatorname{sim}-G A L 4 ; U A S-D^{\text {EnRep }} ; \mathbf{K}\right) \operatorname{sim}-$ GAL4;UAS-mSOX2 ${ }^{\triangle H M G}$; L) sim-GAL4;UAS-mSOx2 ${ }^{\text {EnRep }}$. M-P) Anti-Slit staining to reveal the midline glia. $\mathbf{M}$ and $\mathbf{N}$ ) Wild type at stage 13 (M) and 15 (N). $\mathbf{O}$ and P) sim-GAL4; UAS-D $\triangle H M G$ at stage $13(\mathrm{O})$ and $15(\mathrm{P})$. M and $\mathrm{O}$ are ventral views with anterior to the left, $\mathrm{N}$ and $\mathrm{P}$ are lateral views with anterior to the left. Grey arrowheads indicate loss of Slit expression.
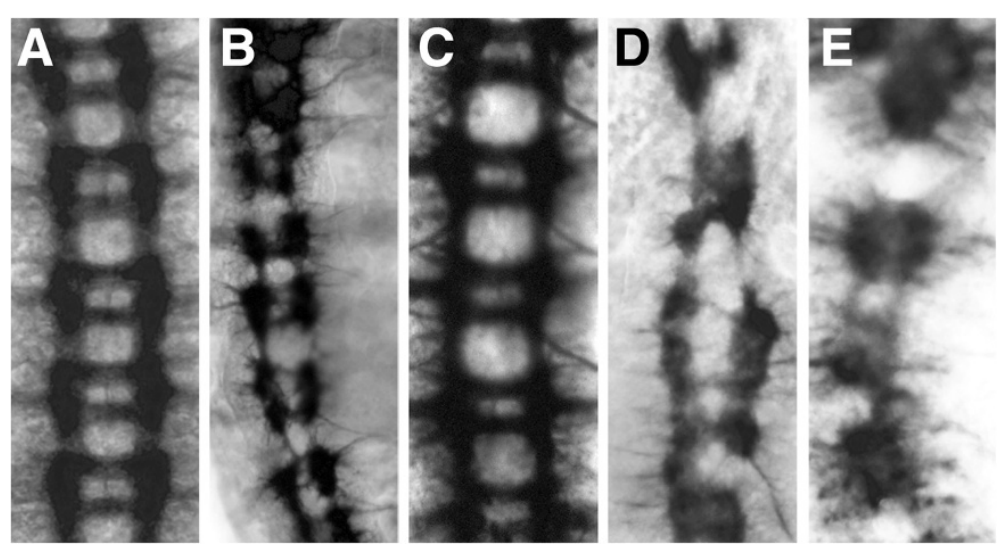

Figure 2 Dominant negative expression in a Dichaete mutant background. A-E) BP102 staining, all embryos oriented with anterior to the

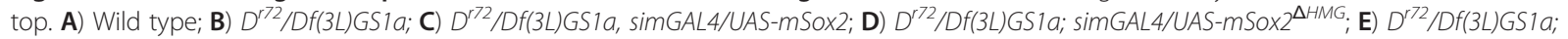
SimGAL4/UAS-mSOX2 $2^{\text {EnRep }}$. 


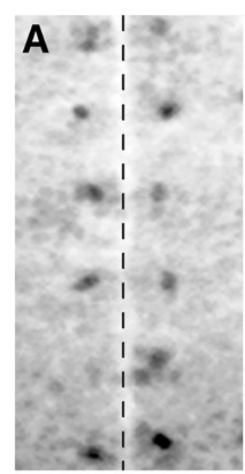

WT

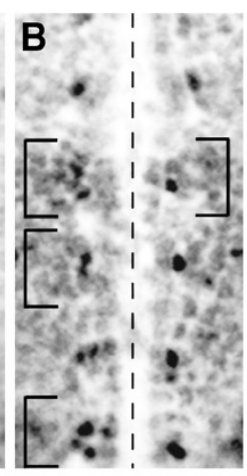

Dichaete

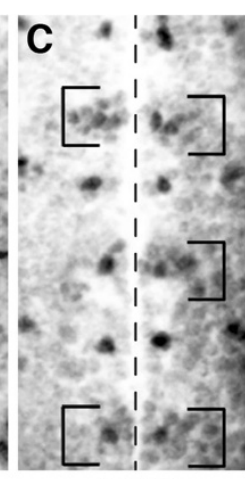

$D^{\triangle H M G}$

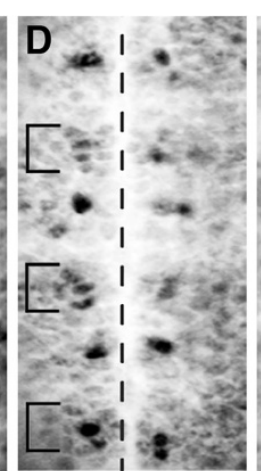

$m$ Sox2 ${ }^{\triangle H M G}$

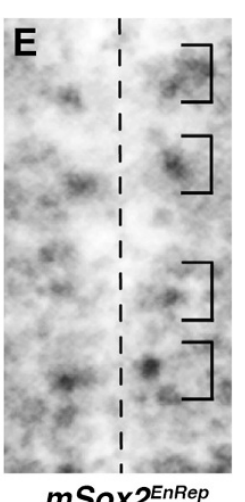

mSox2 EnRep

Figure 3 Proneural gene expression. Ventral views of the medial and intermediate columns of the stage 11 embryonic neuroectoderm stained with anti-Achaete, anterior is to the top. The ventral midline is indicated by the dashed line and neuromeres in individual hemisegments

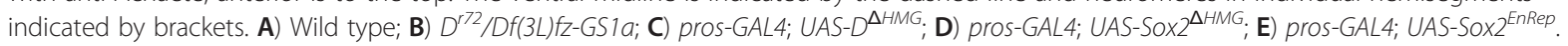

the idea that it interferes with wild type Dichaete function and supports the idea that Dichaete normally acts as a repressor of gene expression in some circumstances.

\section{Dichaete regulates comm in the midline}

To date only two direct Dichaete target genes, slit and $a c$, have been identified and to learn more about how Dichaete regulates gene expression in the developing CNS we sought to identify additional direct Dichaete targets. To do this we examined gene expression data, DNA binding data and the literature to identify candidates genes for further study, focusing on genes known to be expressed in the midline or early neuroectoderm.

Dichaete acts directly in the midline, and in particular is known to function in midline glia $[15,19]$. The commisureless gene is expressed in midline glia [33] and in Dichaete mutants we observe a loss of Comm expression in the midline of stage 12 embryos (Figure 4A and B). Significantly, lateral epidermal stripes of Comm expression as well as earlier blastoderm and neuroectodermal expression (not shown) are not affected in the Dichaete mutants, suggesting Dichaete does not generally regulate comm expression in the embryo.

The hypothesis that comm is a direct target of Dichaete in the CNS was supported by experiments using the DN constructs: expressing $\triangle \mathrm{HMG}$ or EnRep proteins in the midline results in the loss of Comm expression along the length of the midline (50-70\% of embryos, $n>200$ /genotype; Figure $4 \mathrm{C}$ and D). Further to this, strong and ubiquitous expression of mSox $2^{\text {EnRep }}$ via a maternal Gal4-VP16 driver results in a loss of Comm expression in the midline but has little effect on the lateral and early expression of Comm (Figure 4E). This suggests that Dichaete interacts with a specific regulatory element of the comm gene that control midline expression and does not act as a general activator of comm expression. Our observations also further emphasise the functional conservation between Dichaete and Sox2.

Whereas comm expression is lost from the early midline in Dichaete mutant and DN expressing conditions, by stage 14, when high levels of Comm are detected in midline glia and commissural axons of wild type embryos (Figure 4A'), the effects of loss of Dichaete function are less pronounced. While 75-80\% ( $\mathrm{n}>200)$ of the Dichaete$\Delta$ HMG embryos show reduced Comm expression, primarily in midline cells and to a much lesser extent in commissural axons, effects are far less pronounced in Dichaete null mutants or when EnRep constructs are expressed with sim-Gal4 ( 20\% of embryos affected, $n>200$; Figure 4B'-E'). Providing EnRep constructs early

Table 1 Neurectodermal expression of ac under different Dichaete mutant conditions at two different stages of CNS development

\begin{tabular}{lcc}
\hline & Stage 9-10 & Stage $\mathbf{1 1}$ \\
\hline Dichaete $^{-}$ & Intermediate (8\%) & Medial up/extra (42\%) \\
pros-GAL4; UAS-Dichaete $^{\Delta H M G}$ & Intermediate (35\%) & Medial up/extra (25\%) \\
pros-GAL4; UAS-Dichaete $^{\text {EnRep }}$ & Medial down (50\%) & Medial down (ND) \\
pros-GAL4; UAS-mSox2 $^{\text {HHMG }}$ & Intermediate (20\%) & Medial up/extra (42\%) \\
pros-GAL4; UAS-mSOx2 ${ }^{\text {EnRep }}$ & Medial down (45\%) & Medial down (ND) \\
\hline
\end{tabular}

Intermediate $=$ fraction of hemisegments expressing ac in the intermediate column. Medial down = fraction of hemisegments showing loss of ac expression in the medial column. Medial up/extra = fraction of hemisegments showing stronger ac expression or extra ac expressing cells in the medial column. In all cases $\mathrm{n}$ $>40$ embryos. $\mathrm{ND}=$ not done. 

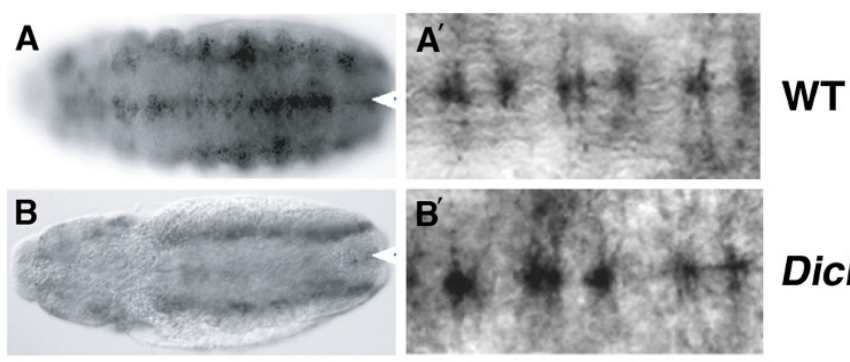

Dichaete
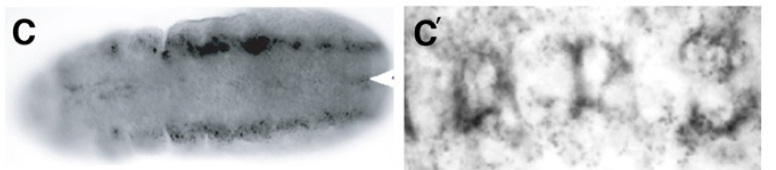

\section{Dim-Gal4
simG}
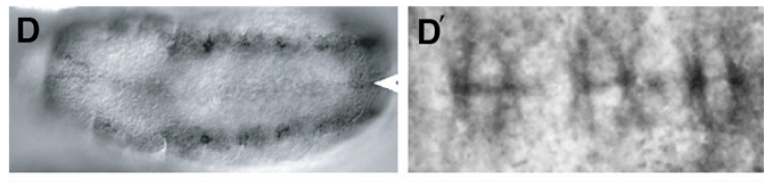

\section{mSOX2EnRep \\ sim-Gal4}
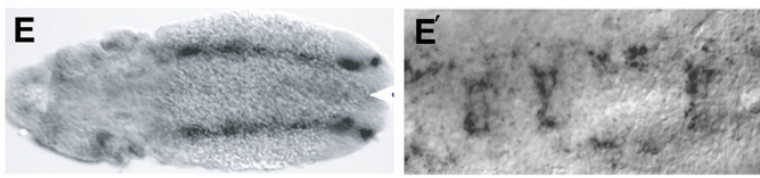

mSox2EnRep
mat-Gal4

Figure 4 Commisureless is a Dichaete target in the midline. Ventral views of stage $12(\mathbf{A}-\mathbf{E})$ and close-up of the midline in stage $14\left(\mathbf{A}^{\prime}-\mathbf{E}^{\prime}\right)$ embryos stained with anti-Comm. Anterior is to the left and the white arrowheads indicate the midline. $\mathbf{A}$ and $\left.\mathbf{A}^{\prime}\right)$ Wild type; $\mathbf{B}$ and $\left.\mathbf{B}^{\prime}\right) D^{r 72} / D f$

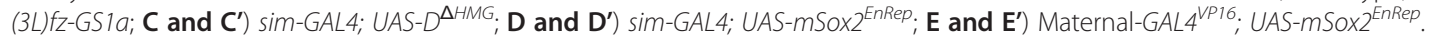

in development via the maternal Gal4 driver results in a loss of Comm in the midline, and again the effects are much less pronounced in axons. Since axonal Comm expression arises from lateral commissural neurons extending processes to the midline, this expression is likely to be regulated by enhancers distinct from those controlling midline expression. We expect that the reduction in axonal Comm expression we observed at the midline is therefore most likely due to a general disruption of the midline by, for example, the loss of Slit. Together our observations suggest that most Dichaete mutant embryos are able to establish glial expression after stage 12 in the absence of Dichaete and suggests that a different, Dichaete independent, regulatory element may control the early and late aspects of comm expression. Taken together with the observation on lateral and early comm expression, our experiments emphasise the modularity of regulatory sequence organisation and suggest that there is little crosstalk between the enhancers controlling specific facets of comm expression.

\section{Dichaete-mediated regulation of ase in neuroblasts}

The expression of the Achaete-Scute Complex (AS-C) genes ac, l'sc and sc in Dichaete and SoxN mutants has already been described $[9,10,16,17]$. However, the effects on the fourth AS-C gene asense (ase) have only been described in SoxN mutants [9], despite also being a potential Dichaete target gene in the developing neuroectoderm.
We therefore examined the effects of Dichaete mutations and $\mathrm{DN}$ constructs on its expression.

In the wild type embryo, ase is initially expressed in CNS neuroblasts in three well-defined columns at late stage 9 [34]. In Dichaete null mutant embryos (Figure 5A-J), the expression of Ase is detected earlier and is stronger at stages 9 and 10 than in wild type. After stage 10, the intensity of Ase expression shows no apparent difference between wild type and Dichaete mutants. At later stages, the Ase expression pattern becomes disrupted, reflecting, at least in part, the segmentation and CNS disruptions characteristic of Dichaete mutants. Thus, as with $a c$ in the intermediate column, Dichaete appears to act as a repressor of ase. However, in contrast to the results with $a c$, the effects on ase expression differ between Dichaete mutants and the DN-Sox constructs. With Dichaete ${ }^{\Delta H M G}$ driven by pros-Gal4, we observe a general loss of the Ase expressing cells in neuroectoderm in 10-15\% of embryos, particularly obvious in the medial and intermediate columns after stage 11 (Figure $5 \mathrm{~K}$ and L). Embryos expressing the other Sox DN constructs show much less severe phenotypes.

\section{Dichaete binding at target genes}

To strengthen the view that Dichaete directly regulates comm and ase, we examined the genome-wide ChIParray binding profiles generated by BDTNP [23] and the modENCODE project [24] and found binding intervals 


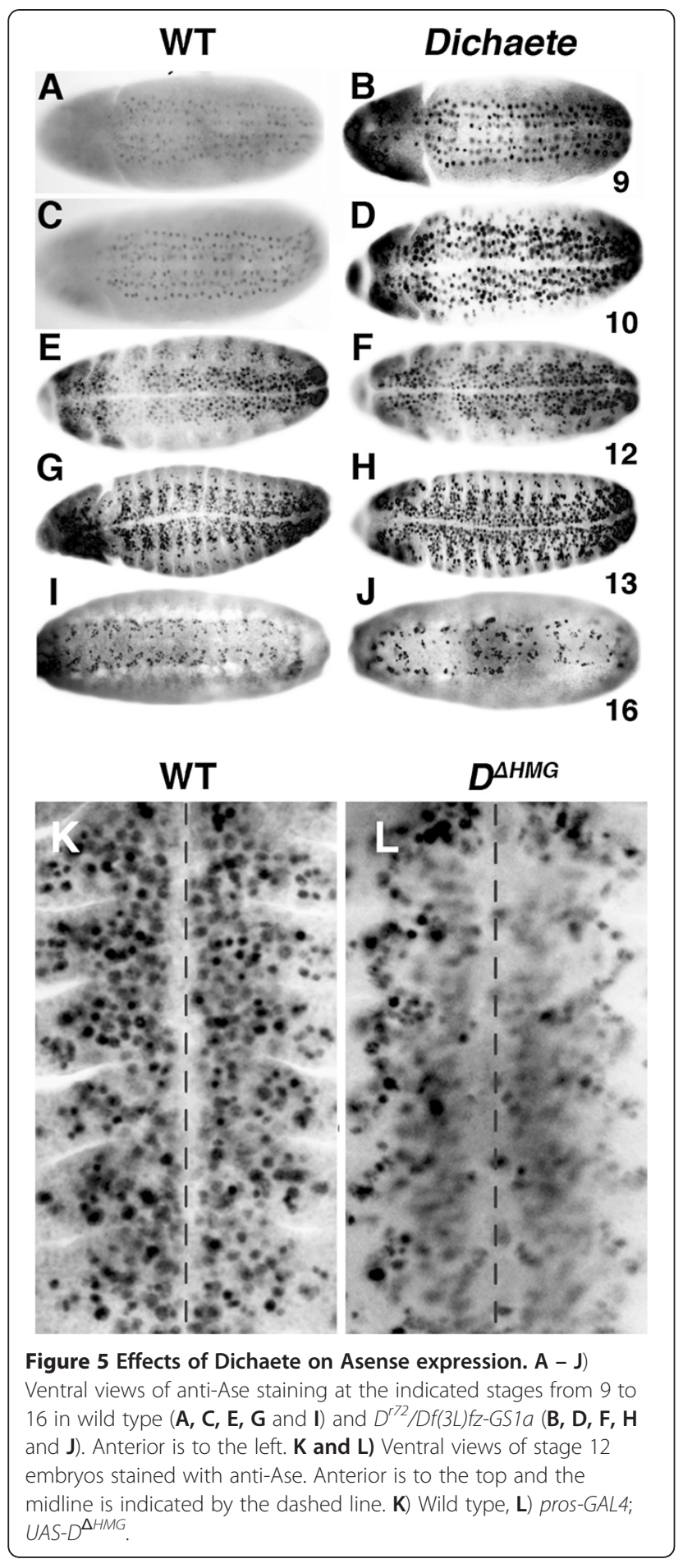

identified at $1 \%$ false discovery rate (FDR) cut-offs. The BDTNP data were generated using chromatin from 2-3 hr (stage 5) embryos and the modENCODE data from 0-8 hr (stage 1-12) embryos. Together these data may not entirely reflect aspects of late Dichaete binding. We therefore performed specific ChIP-PCR assays around the ase and comm genes as well as the known target sli using chromatin extracted from $0-12 \mathrm{hr}$ embryos (up to stage 14). In the case of $s l i$, the BDTNP data shows 6 high confidence binding intervals distributed across various introns and we assayed two of these regions with a series of ChIPPCR assays (Figure 6A). Using a series of 28 primer pairs encompassing a region around the known midline enhancer [19] and a region spanning the 5 ' end of the gene, we find evidence for extensive Dichaete binding that is as broad, if not broader, than the binding regions identified using ChIP-array. Encouragingly, the strongest enrichment was observed with primers covering the known midline enhancer (fragment 13). In the case of ase, the BDTNP data identified four regions, two 5 ' and two 3 ' to the transcription unit. We found the strongest ChIP-PCR signals from primers encompassing the immediate 3 ' region (fragments 13-16; Figure 6B) but also found weak signals across the body of the genes. In the case of comm, we found the strongest ChIP-PCR signals at the immediate 5' end of the gene (fragments 12 \& 13) and also found evidence for Dichaete binding further 5, partially overlapping a region identified in the BDTNP and modENCODE data (fragments 15-18; Figure 6C). While there is agreement between the ChIP-array and PCR-based assays, there are clearly some differences. The BDTNP data from the blastoderm stage appears to identify much broader binding than modENCODE, and we note that we see evidence for considerable low level amplification in the PCR assays, with many fragments producing weak but detectable products in comparison to the pre-immune control. These differences may simply reflect the different embryonic stages used in each study or reflect differences in crosslinking conditions. While higher resolution ChIP-seq data will be required to adequately define the extent of Dichaete binding at particular regulatory elements, our experiments confirm that Dichaete is directly associated with the potential targets genes.

Taken together with the evidence from immunohistochemistry experiments on the potential targets in Dichaete mutants and DN conditions, these data strongly suggest that ase and comm are direct Dichaete targets in the embryonic CNS. Interestingly, the Ascl1 and Slit1 genes in mouse, which are orthologous to ase and sli respectively, are associated with Sox 2 binding [35], hinting that, along with the functional conservation displayed by Dichaete and Sox2 [15], some of their genetic networks in neural precursors may also be conserved.

\section{Tissue-specific expression profiling}

While candidate gene approaches are useful for identifying a small number of target genes, genomics methods can offer a more global perspective. To this end, we used the dominant negative constructs to perform genomewide gene expression studies to identify a broader range of potential Dichaete target genes in specific subsets of 


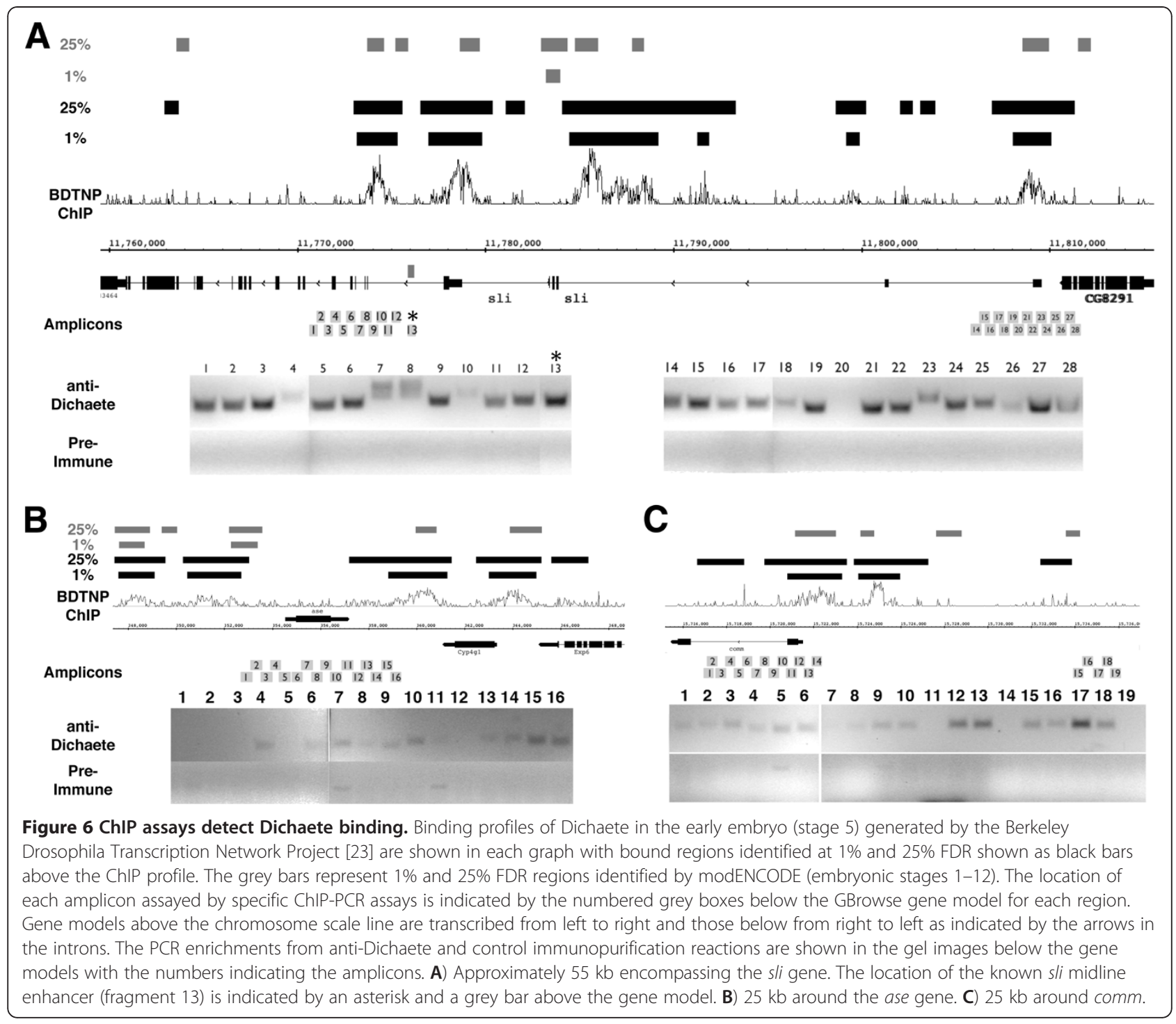

the CNS. Microarray gene expression analysis was performed with RNA extracted from whole stage 9 embryos expressing the Dichaete DN constructs in the ventral midline or in neuroblasts; the wild-type construct was also included to identify any effects of Dichaete overexpression in the tissues in question. Each construct was expressed in the midline with sim-Gal4 and in neuroblasts with pros-Gal4, and four biological replicates were performed for each experiment. A UAS- $g f p$ construct was crossed to both Gal4 drivers in parallel with the Dichaete lines to provide control embryos. Using our standard microarray analysis, a number of differentially expressed genes were detected (Table 2).

Across the six microarray studies, we found a total of 994 genes with a significant change in expression in at least one of the experiments. Focusing first on the ventral midline, 214 genes in total changed expression in at least one of the experiments driving a Dichaete construct with sim-GAL4 (Additional file 1), with the $\triangle \mathrm{HMG}$ construct showing a stronger effect (169 genes) than the EnRep (48 genes) or wild type (45 genes) constructs. A total of 11 genes are common to all three experiments, and 8 are common to both $\mathrm{DN}$ expressing conditions (Figure 7A), including bancal, photorepair and Cyp4p2, which are downregulated. The gene bancal was flagged as a target in all 6 gene expression studies, the BDTNP ChIP data shows Dichaete binding around it, and it is annotated with gene ontology (GO) terms "transcription factor binding" and "mRNA binding", as well as "cell proliferation" and "cell fate commitment", making it an interesting potential target of Dichaete. Myosin61F, Ras-related protein (Rala) and Cap-H2 are upregulated, suggesting they may be indirect targets. While we did not identify a significant change in sli expression, we did find 6 genes identified in a screen for midline expressed genes, including kek1, $K r-h 1$, Sema-5c and nemy [36]. The comparatively small 
Table 2 Number of upregulated and downregulated genes detected by microarray analysis using the indicated genotypes

\begin{tabular}{lccc}
\hline \multicolumn{1}{c}{ Genotype } & Upregulated & Downregulated & Total \\
\hline sim-GAL4; UAS-Dichaete $^{\text {UHMG }}$ & 50 & 119 & 169 \\
sim-GAL4; UAS-Dichaete $^{\text {EnRep }}$ & 13 & 35 & 48 \\
sim-GAL4; UAS-Dichaete $^{W T}$ & 17 & 28 & 45 \\
pros-GAL4; UAS-Dichaete $^{\text {AHMG }}$ & 66 & 533 & 599 \\
pros-GAL4; UAS-Dichaete $^{\text {EnRep }}$ & 119 & 154 & 273 \\
pros-GAL4; UAS-Dichaete $^{\text {WT }}$ & 18 & 66 & 84 \\
\hline
\end{tabular}

The thresholds used to identify differentially expressed genes were average M-value of $<-0.5$ or $>0.5, p$-value $<0.05$.

number of gene expression changes we identified is likely to be a reflection of the fact that the ventral midline is a very small component of the embryo and thus only very pronounced changes in gene expression are expected to be detected by the microarray analysis.

In the neuroblast experiment, driving the $\mathrm{DN}$ constructs with pros-GAL4, we identified a total of 851 genes that changed expression in at least one of the experiments (Figure 7A; Additional file 2). It was not unexpected that this experiment affects a larger number of genes since the tissue targeted is larger than the midline and consequently it is easier to pick up more subtle changes in gene expression. Again the $\triangle \mathrm{HMG}$ construct showed the strongest effect (599 genes) with a smaller number of changes detected when expressing for the EnRep (273 genes) or wild type (84 genes). While we might have expected the EnRep construct to consistently downregulate genes, we were surprised to find that $30-40 \%$ of misregulated genes showed significant upregulation (Figure 7B; Table 2). Focusing on the genes changing expression in both the $\mathrm{DN}$ conditions, we identified 80 genes and of these 74 were downregulated in the presence of the EnRep construct. We found that 15 of these have GO annotations associated with proteolysis and while we considered that this could be a stress or cellular damage response, we note that 14 of these genes were downregulated by the expression of the $\triangle$ HMG construct, suggesting they require Dichaete function for their activation.

We identified 69 genes that changed expression in both the midline and the neuroblast experiments, suggesting they are good candidate target genes. While we did not identify any significantly enriched gene ontology terms associated with this set of genes, we did find 30 terms linked to development with corrected p-values lower than

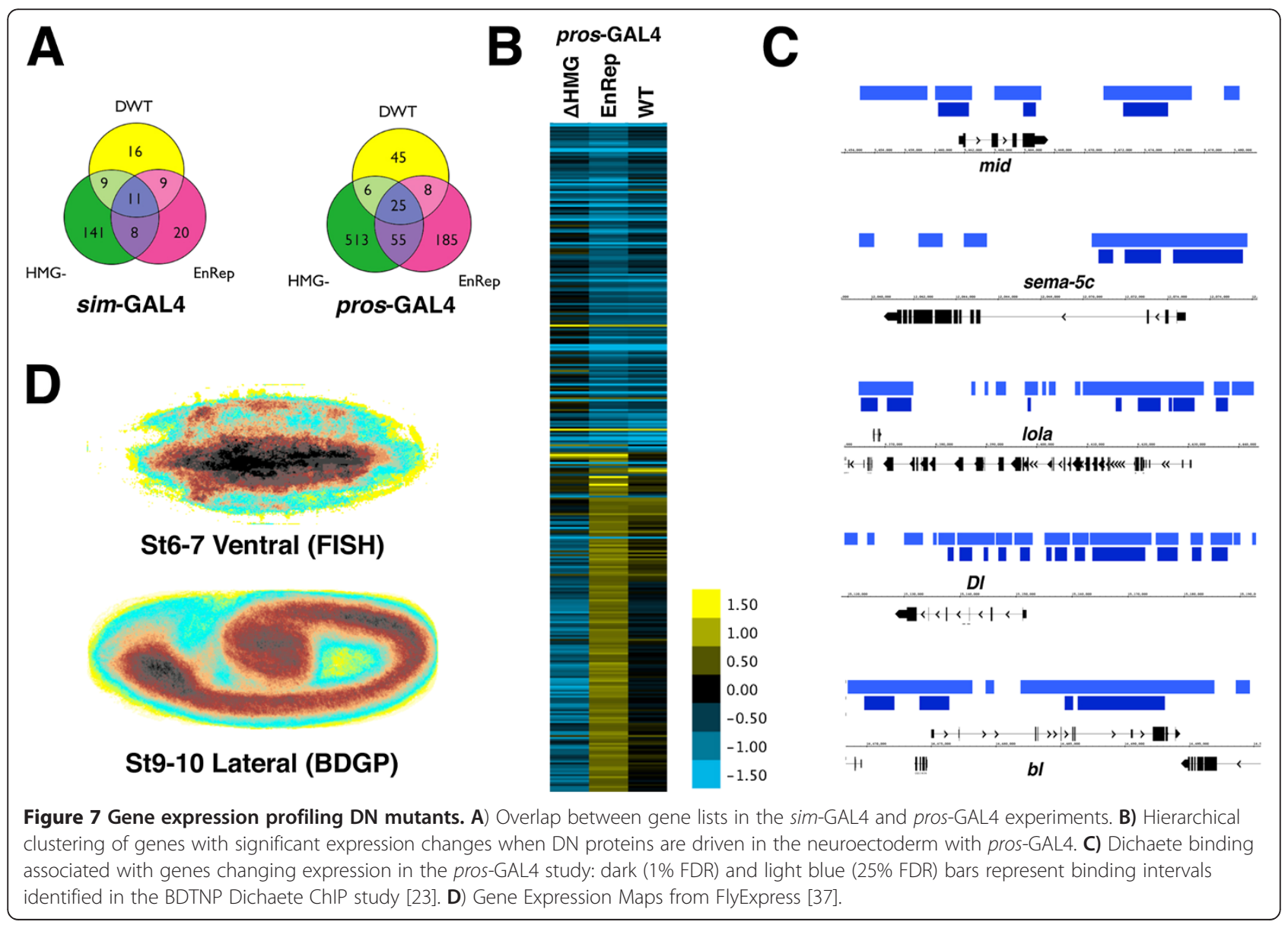


0.01 when we examined the overall list of list of 994 differentially regulated genes (Additional file 3). To find further evidence supporting direct Dichaete regulation, we compared the set of 994 genes with published genome-wide ChIP datasets [23,24] and found that 503 (51\%) of these have evidence for Dichaete binding, suggesting that a substantial fraction of the genes we identify may be genuine Dichaete targets (Figure 7C, Additional file 4). Significantly, when we examined the list of 503 genes showing expression changes and evidence of Dichaete binding in the ChIP study, 60 development related ontology terms were found with much lower $\mathrm{p}$ values $(1.00 \mathrm{E}-$ 09 ), including ontology terms associated with nervous system development (GO:0007399) and generation of neurons (GO:0048699).

Of these 503 Dichaete bound genes, 107 have in situ based gene expression data available in the FlyExpress database [37]. Creating a Gene Expression Map from these genes shows an enrichment in the ventral neuroectoderm and midline early in embryogenesis, and very strong CNS enrichment at Stage 9-10, when the gene expression profiling was performed (Figure 7D). This suggests that the genes showing expression changes and Dichaete binding are likely to constitute bona fide Dichaete targets. We submitted the list of Dichaete bound genes to i-cisTarget [38] to search for enriched transcription factor binding site matrices and identified a Dichaete-like AACAA motif (a consensus Dichaete binding motif is AACAAT [39]) associated with $45 \%$ of the genes (11th of 127 enriched motifs, e-score $=4.12$ ). Interestingly, we also found enrichment for motifs associated with TFs identified as potential Dichaete targets (Lola, $\mathrm{e}=3.22 ; \mathrm{Hb}, \mathrm{e}=2.82$ and $\mathrm{E}(\mathrm{bx}), \mathrm{e}=2.76$ ), hinting at potential regulatory interactions.

These new Dichaete target genes include a number of transcriptional regulators (e.g. achaete, bunched, d4, E2f, hunchback, huckebein, longitudinals lacking, midline, mushroom body defective, p53 and SoxN) as well over 100 genes annotated with the GO term signalling, including several components of the Notch (e.g. Delta, Hairless, Notchless, bunched), Egfr (Cbl, csw, sno, sty), Wnt ( $f z, f z 3, n k d$, pygo) and JAK-STAT (hop, Stat92E) pathways. We noticed that 20 of the new target genes are annotated as being involved in lateral inhibition, further implicating Dichaete in the early events in the neuroectoderm. If Notch signalling is compromised in the early neuroectoderm, lateral inhibition breaks down and excess neural cells are produced at the expense of epidermis [40]. In our analysis of $a c$ expression we found extra Ac-positive cells in the medial column consistent with a reduction in lateral inhibition (Figure 3). In particular, we noticed that while expression of EnRep constructs generally results in loss of $a c$ expression, we did observe hemisegments with increased numbers of $a c$-positive cells (Figure 3E). As well as these early events in neural development, we found 50 genes involved in neuron differentiation (i.e. axonogenesis, axon fasciculation and guidance, dendrite morphogenesis), suggesting that Dichaete acts at the level of terminal differentiation genes.

Focusing on aspects of neural development, we found that $18 \%$ (89 genes) of the bound and mis-regulated genes have $\mathrm{CNS}$ development GO annotations (corrected p-value $=0.001$ ). Analysis of this set of 89 genes with the STRING interaction database [41] reveals a highly connected network centred on the Wnt and Egfr pathways, further emphasising the Dichaete role in regulating multiple signalling pathways during nervous system development (Additional file 5). Taken together, our expression analysis combined with published Dichaete binding data identify a large number of likely direct targets. Clearly these genes will need further validation, however the lists provide a platform for the further analysis of group B Sox activity in subsets of the CNS. Our experiments also emphasise that ideally data from multiple experiments is needed when interpreting results generated by experiments expressing dominant negative proteins due to the lack of sensitivity and existence of off-target effects.

\section{Discussion}

In this study we have investigated the use of dominant negative alleles of the Sox domain transcription factor Dichaete to identify tissue-specific functions and potential target genes. We show that expression of Dichaete alleles with a deleted DNA binding domain or fused to a strong transcriptional repressor in subsets of the embryonic CNS produce Dichaete-like phenotypes. Analysis of predicted target genes in the CNS midline (comm) or neuroectoderm (ase) suggest that Dichaete directly regulates these genes as judged by molecular phenotypes observed both in Dichaete mutants and when exposed to the DN constructs. Expression profiling embryos expressing DN constructs in the CNS midline or in neuroblasts identifies approximately 1000 genes showing altered expression. While some of the identified gene expression changes will be attributable to off-target effects of the DN constructs, we were encouraged to find that half of misexpressed genes are associated with in vivo Dichaete binding, indicating that we have identified a substantial repertoire of new Dichaete targets.

We validated the activity of the DN constructs on the established Dichaete midline target gene slit, showing that expression of both proteins in the midline led to loss of slit expression and Dichaete-like midline phenotypes. Similarly, in the neuroectoderm we confirmed previous work showing that $a c$ is a direct Dichaete target $[16,17]$ with the $\triangle \mathrm{HMG}$ construct leading to derepression of $a c$ in the medial column of the neuroectoderm, expression of the EnRep fusion leading to $a c$ repression 
and ChIP-PCR assays with Dichaete antiserum enriching regulatory sequences in the slit gene.

We went on to identify two new Dichaete targets in the CNS, comm in the midline and ase in the neuroectoderm. In midline glia, a cell type where Dichaete is known to control slit expression, the comm gene is also expressed in the midline although its function here is not understood [42]. Targeting DN expression to the midline, we found a loss of Comm expression. Significantly, we also found that when EnRep fusions were expressed throughout the embryo via a maternal GAL4 driver, only midline Comm expression was affected while lateral domains were unaffected. These observations indicate that the action of DN Dichaete constructs on comm is not a general effect, but instead targets specific regulatory elements. Again we found support for direct Dichaete action by analysing published Dichaete ChIParray data and performing specific ChIP-PCR assays.

These observations suggest that Dichaete is only involved in the initial establishment of comm expression in the midline, since later in development comm becomes Dichaete independent. This is analogous to our previous finding that Dichaete is required for the establishment of primary pairrule gene expression at the blastoderm stage, but that after cellularisation these genes are expressed independently of Dichaete [5]. This difference between expression establishment and maintenance has previously been described for the Runt repressor and may be a more widespread feature of transcription factor biology [43]. However, arguing against Sox-independent late comm expression, we note that stage 12, when comm expression recovers in Dichaete mutants, is when the SoxN gene first becomes active in the midline [9], suggesting loss of Dichaete may rescued by SoxN expression. Since we find that late comm expression is affected in $\triangle H M G$ expressing embryos and when the EnRep fusions are supplied ubiquitously, it is possible that Dichaete and SoxN use the same co-factors to regulate comm.

In the neuroectoderm we found that ase, a member of the AS-C that acts as a neural precursor gene [34,44], is derepressed in Dichaete mutants. We found earlier than normal expression of ase in the early neuroectoderm along with evidence for Dichaete binding in ChIP assays. Thus, like the proneural AS-C complex member $a c$, Dichaete appears to act as a repressor. Whether this is also in conjunction with the Ind and Vnd homeodomain proteins remains to be determined. Interestingly, in Dichaete mutants we observe derepression of ase in all three columns of the neuroectoderm, whereas Dichaete expression is restricted to the medial and intermediate columns after gastrulation. This observation suggests that earlier Dichaete expression at the blastoderm stage may influence prepatterning throughout the neuroectoderm. We expect that expression of the EnRep constructs would have little effect at stage $9-10$ since ase expression is already very low in the wild type at this stage. It was also observed that expression of $\triangle$ HMG constructs has little effect during early stages of development. There are a number of possible reasons for this: most likely Dichaete mediated ase repression occurs earlier in development than the activation of the pros-Gal4 driver, alternatively the $\triangle \mathrm{HMG}$ constructs might be unable to sequester the relevant co-factors. It is also possible that ase may be an indirect target, however our other evidence suggests this is not the case. We found severe disruption of ase expression when all of the DN constructs are expressed via a maternal Gal4 driver (not shown), however it is likely that this is due to pleiotropic effects of inhibiting Dichaete function.

Our analysis uncovered a number of potential off-target effects resulting from the expression of both versions of DN Dichaete. In particular we note that phenotypes tended to be stronger than those observed in Dichaete null embryos and we also saw stronger phenotypes when expression of DN constructs was performed in a Dichaete null background. We do not attribute these effects to misexpression per se, since expression of wild type Dichaete under the same conditions resulted in very mild phenotypes and also rescues Dichaete null phenotypes. In addition, expression of wild type Dichaete resulted in a small number of significant gene expression changes, presumably reflecting some dosage-dependent effects of Dichaete. Unexpectedly from the phenotypic analysis, we found that both in the midline and in neuroblasts the $\triangle H M G$ construct affected the expression of twice as many genes as the EnRep construct. Expression of $\triangle \mathrm{HMG}$ mostly led to down regulation of transcript levels, with $70-90 \%$ of significantly changed genes repressed compared to controls. We imagine that the $\triangle \mathrm{HMG}$ protein acts by sequestering DNA binding proteins or other cofactors that normally interact with Dichaete but that this will also result in these cofactors being unavailable for interaction with other TFs and thus affect other, Dichaete-independent, processes. For example, we know that Dichaete interacts with Vvl and Sim in the midline $[15,19]$, however, both these proteins play much more extensive roles in embryonic development and, in particular, show stronger midline phenotypes than Dichaete mutants [45,46]. Similarly, Dichaete is known to interact with Ind $[16,47]$ in the neuroectoderm, but only in a subset of this transcription factor's expression domain. Therefore at least some of the effects we see from expression of the $\triangle \mathrm{HMG}$ will be attributable to direct action on Dichaete targets.

In the case of the EnRep fusion, we anticipated that binding of this protein to Dichaete targets in the genome would result in the repression of these genes [30], a view reinforced by preliminary data from expression profiling Dichaete null mutant embryos indicating that Dichaete mainly acts as a transcriptional activator (JA and SR, unpublished observations). We were therefore surprised to 
find as much as $40 \%$ of misregulated genes identified in the EnRep screens showed increased expression compared to controls. These observations may indicate a substantial level of off-target effect, where upregulated genes reflect secondary changes in expression due to repression of a gene encoding a repressor. Alternatively, the upregulation may result from unanticipated interactions between the regulatory proteins binding together at cis-regulatory elements such that the effects of the EnRep domain are masked. Finally, it is possible that in the case of some highly expressed genes the Engrailed Repressor domain may not function and the EnRep construct behaves like a Dichaete overexpression construct.

While our focused analysis identified two likely Dichaete targets, the published ChIP data and our preliminary Dichaete mutant expression profiling suggest that Dichaete may regulate thousands of targets in the Drosophila genome, and the expression profiling experiments presented here support this view. While some of the gene expression changes we identify reflect secondary consequences downstream of direct Dichaete targets, we were reassured to find evidence for Dichaete binding associated with over half of the misregulated genes. Significantly, these bound and regulated genes are enriched for nervous system expression and also show significant over-representation of gene ontology terms associated with development and specifically CNS development. A number of genes encoding transcription factors associated with CNS development were identified in the screen (achaete, bunched, d4, E2f, hunchback, huckebein, longitudinals lacking, midline, mushroom body defective, p53 and SoxN), indicating that Dichaete may play diverse roles in controlling aspects of the regulatory networks underpinning CNS development. We also identified enriched binding motifs for $\mathrm{Hb}$ and Lola associated with these bound and regulated genes, hinting at potential regulatory interactions involving Dichaete. Of particular interest is $\mathrm{Hb}$, which is known to be involved in the temporal sequence of TFs that specify neuroblast fates [48]. Dichaete has been shown to participate in a similar process during larval CNS development [22] and our observations suggest that, as well as being expressed together in neuroblasts, there may be a regulatory loop between Dichaete and $\mathrm{Hb}$ in the embryo.

Other potentially interesting Dichaete targets include nejire (nej), encoding a histone acetyltransferase involved in both segmentation and neuron differentiation, bancal, encoding a splicing regulator, and several components of the Notch signalling pathway including the genes encoding the Delta ligand and the transcriptional co-repressor Hairless. As we note above, the presence of extra $a c$ expressing cells observed when expressing DN constructs is consistent with a loss of lateral inhibition and suggests that Dichaete regulates multiple steps in the neural specification pathway. Group B Sox proteins have previously been shown to regulate Notch signalling in vertebrates by directly regulating the Hes class bHLH genes [28,49] and we identified the Notch pathway repressor encoded by Hairless as well as the Bearded-family member $E(s p l) m 4$ as putative direct Dichaete targets. We also identified several components of the Wnt signalling pathway as target genes. Previously both Dichaete and SoxN have been shown to negatively regulate the Wnt pathway in the embryonic epidermis [50,51] and our studies suggest this extends to other tissues. Interestingly, in the epidermis a key target of SoxN/Dichaete is shavenbaby, which is regulated by both Wnt and EGFR signalling. Our screen also identified sprouty and $C b l$, encoding two global inhibitors of the EGFR pathway [52], suggesting a more general Soxmediated interaction between EGFR and Wnt pathways. Sox factors have been proposed to interact with Wnt signalling pathways during a variety of developmental processes [53] and our data suggests that this may occur at multiple levels.

\section{Conclusion}

Taken together our study acts as a starting point for further analysis of the role Dichaete plays in the gene regulatory networks governing the development of the embryonic CNS in Drosophila. We validate new Dichaete target genes in the midline and neuroectoderm as well as identifying a set of over 500 Dichaete bound and regulated genes. Given the functional conservation between Dichaete and vertebrate Sox2, as well as the wider association between group B Sox proteins and CNS development, we suggest these data may be more widely applicable to understanding the role Sox proteins play in critical developmental processes.

\section{Methods \\ Drosophila stocks}

All fly stocks were maintained on standard yeast-cornmeal media at $25^{\circ} \mathrm{C}$. The following stocks were used (all nomenclature is according to FlyBase): $D^{r 72} / T M 6 B, D f(3 L) f z$ GS1a/TM3 Sb ${ }^{1}$, w; p $\left\{\right.$ GAL4-pros $\left.{ }^{C 20}\right\}$ C74-17,w; p\{GAL4sim3.7\}2/CyO; p\{GAL4-sim3.7\}3, w; p\{mato4-GAL-VP16\} $V 2 H, w ; p\{m a t \alpha 4-G A L-V P 16\} V 37$.

\section{Generating dominant negative constructs}

To generate pUAST-Dichaete ${ }^{\Delta H M G}$, pUAST-Dichaete ${ }^{\text {EnRep }}$ and pUAST-Dichaete constructs, cDNA fragments were amplified from a cDNA clone [5] by standard PCR using PfuTurbo DNA polymerase (Stratagene) and cloned into the pBluescript II KS (-) vector. For the Dichaete ${ }^{\Delta H M G}$ construct, an $\mathrm{N}$ terminal fragment encompassing amino acids 1 to 135 was amplified with primers containing a 5' EcoRI site and a 3' XhoI site, cut with EcoRI and XhoI, and cloned into pBluescript II KS(-). The C-terminal Dichaete fragment, from amino acids 210 to 282 . was amplified with 
primers containing a 5' XhoI site and a 3' KpnI site, cut with $X h o I$ and KpnI, and cloned into the plasmid containing the N-terminal fragment. For the full-length Dichaete construct, the insert was amplified from the full-length cDNA clone (GenBank accession X96419 and cloned into the HindIII and KpnI sites of pBluescript II $\mathrm{KS}(-)$. For the Dichaete ${ }^{\text {EnRep }}$ construct, the Engrailed repressor domain (amino acids 1-298) was amplified with primers containing a 5' EcoRI site and a 3' HindIII site (Engrailed cDNA kindly provided by B. Sanson, University of Cambridge), cut with EcoRI and HindIII, and cloned into the pBluescript II $\mathrm{KS}(-)$ vector containing the full-length Dichaete cDNA. Subsequently, Dichaete fragments were amplified from the pBluescript plasmids with PfuTurbo polymerase and cloned into the $\mathrm{pENTR}^{\mathrm{TM}}$ / D-TOPO vector (Invitrogen) with a Directional TOPO Cloning Kit (Invitrogen). The TOPO entry clones were used to recombine the Dichaete fragments in-frame with a C-terminal GFP cassette in a pUAST Gateway destination vector, pUAST-attR1-CmR-ccdB-attR2-cGFP (generated by the Martinez Arias Laboratory, Department of Genetics, University of Cambridge). The UAS mouse Sox2, UAS mouse So $x 2^{\triangle H M G}$ and UAS mouse Sox $2^{\text {EnRep }}$ constructs were kindly provided by Dr. Karine Rizzoti (NIMR, Mill Hill). The UAS mSox2 construct contains a full-length wild type mouse Sox 2 cDNA fused with a C terminal EYFP tag. The UAS So $x 2^{\triangle H M G}$ construct contains a C-terminal fragment of the mouse Sox 2 cDNA fused with a $C$ terminal EYFP tag followed by a Myc tag. UAS $\operatorname{Sox} 2^{\text {EnRep }}$ contains an N-terminal fragment of mouse Sox2, including the HMG domain, fused to the Engrailed repressor domain at the $\mathrm{C}$ terminus followed by a Myc tag. Transgenic flies were generated by standard germline transformation [54].

\section{Immunohistochemistry}

Embryos were collected from yeasted apple juice agar plates, washed thoroughly with water and dechorionated in $50 \%$ commercial bleach for $3 \mathrm{~min}$. Dechorionated embryos were fixed and processed for immunohistochemistry with the Vector $\mathrm{ABC}$ Elite $\mathrm{Kit}^{\mathrm{TM}}$ (Vector laboratories) using minor modifications to standard techniques [55]. Stained embryos were mounted in $70 \%$ and examined with a Zeiss Axiophot microscope using bright-field or Nomarski optics. Images recorded with a digital camera using Openlab software and processed with Adobe Photoshop. The following antibodies were used at the stated dilutions: mouse antiAchaete (1:5, Developmental Studies Hybridoma Bank), rabbit anti-Asense (1:10, gift of A. Brand), Rabbit antiCommisureless (1:50 gift of G. Tear), Mouse anti-Fasciclin II (1:4, Developmental Studies Hybridoma Bank 1D4), mouse anti-Slit (1:10, Developmental Studies Hybridoma Bank C555.6D).

\section{ChIP assays}

Chromatin immunopurification from Drosophila embryo collections using Rabbit anti-Dichaete [15] or control IgG was carried out as previously described [56]. Immunopurified chromatin from anti-Dichaete and control reactions was assayed in parallel with oligonucleotide primer pairs that amplify fragments of the regulatory regions of ase, comm and slit (Primer sequences designed with Primer 3). ChIP-array data from [23] was obtained from Array Express (E-TABM-736) and from the modMINE website (E08_D | modENCODE_2571) [57]. Binding intervals were visualized using the Integrated Genome Browser [58]. Comparisons between expression data and binding data were made via custom lists in FlyMine [59].

\section{Microarray analysis}

Approximately 150 embryos per sample were collected between 3.5 and 4.5 hours after egg laying and stored frozen in Trizol. Following RNA extraction, reverse transcription, Klenow amplification and labelling, samples were hybridised to INDAC FL002 (GEO: GPL5016) or FL003 (GEO:GPL14121) Drosophila gene expression arrays using our standard protocols (http://www.flychip.org.uk/). Four biological replicates were performed for each experiment, with 2 dye swaps incorporated into the experimental design to control for bias due to different dye incorporation efficiencies. Arrays were quality checked manually, removing any spots that appeared to be affected by high levels of background or artefacts. Our standard data analysis pipeline was employed (http://www.flychip.org.uk/) using Dapple for spotfinding and quantifying signal intensities [60], and the Variance Stabilisation and Normalization (vsn) package in $R$ [61] followed by CyberT [62] to assess statistical significance. The thresholds used to identify differentially expressed genes were average $\mathrm{M}$-value of $<-0.5$ or $>0.5$ with a $\mathrm{p}$-value $<0.05$.

\section{Additional files}

Additional file 1: Table of significant gene expression changes observed driving DN Dichaete constructs in the midline with simGAL4. Transcript = FlyBase designated transcript against which the array probe was designed. Gene = FlyBase gene name. $M=$ average log2 fold change. $p=p$ value.

Additional file 2: Table of significant gene expression changes observed driving DN Dichaete constructs in neuroblasts with prosGAL4. Transcript = FlyBase designated transcript against which the array probe was designed. Gene= FlyBase gene name. $M=$ average log2 fold change. $p=p$ value.

Additional file 3: Table of enriched Gene Ontology terms associated with a) the list of all 994 genes changing expression across the microarray studies and b) the subset of 503 genes with associated Dichaete binding.

Additional file 4: Table of genes with DN-Dichaete dependent expression changes and associated Dichaete binding intervals. 
Additional file 5: Figure showing the output from the STRING database using the 89 Dichaete target genes with Nervous System Development annotations (GO:0007399). In this network the thickness of the edges represents the confidence in the interaction, with thicker lines showing stronger associations. All nodes show Dichaete binding and expression changes apart from those highlighted: ** Dichaete binding and expression change below significance cut-off, * Dichaete binding only.

\section{Competing interests}

The authors declare that they have no competing interests.

\section{Authors' contributions}

SPS generated the dominant negative lines, carried out the phenotypic analysis and performed the ChIP-PCR assays. JA performed the microarray and genomics analysis, and helped write the manuscript. SR designed the experiments, analysed data and wrote the manuscript. All authors read and approved the final manuscript.

\section{Acknowledgements}

We are indebted to K. Risotti and R. Lovell-Badge for the mouse Sox2 constructs. We thank B. Sanson, A. Martinez Arias, A. Brand and G. Tear for reagents; $B$. Fischer for assistance with the microarrays and J. Roote for assistance with the flies. We thank the reviewers for their helpful comments on the original version of the manuscript. This work was supported by an MRC research studentship to JA and an MRC programme grant to SR and M. Ashburner. All authors read and approved the final manuscript.

\section{Author details}

'Department of Genetics, University of Cambridge, Cambridge, UK. ${ }^{2}$ Cambridge Systems Biology Centre, University of Cambridge, Cambridge, UK. ${ }^{3}$ Present address: Wellcome Trust Sanger Institute, Wellcome Trust Genome Campus, Hinxton, UK.

Received: 24 October 2012 Accepted: 3 January 2013

Published: 5 January 2013

\section{References}

1. Kalinka AT, Varga KM, Gerrard DT, Preibisch S, Corcoran DL, Jarrells J, Ohler $U$, Bergman CM, Tomancak P: Gene expression divergence recapitulates the developmental hourglass model. Nature 2010, 468:811-814.

2. Masui S, Nakatake Y, Toyooka Y, Shimosato D, Yagi R, Takahashi K, Okochi H, Okuda A, Matoba R, Sharov AA, et al: Pluripotency governed by Sox2 via regulation of Oct3/4 expression in mouse embryonic stem cells. Nat Cell Biol 2007, 9(6):625-635.

3. Blanco J, Girard F, Kamachi Y, Kondoh H, Gehring WJ: Functional analysis of the chicken delta1-crystallin enhancer activity in Drosophila reveals remarkable evolutionary conservation between chicken and fly. Development 2005, 132:1895-1905.

4. Nambu P, Nambu J: The Drosophila fishhook gene encodes a HMG domain protein essential for segmentation and CNS development. Development 1996, 122:3467-3475.

5. Russell SRH, Sanchez-Soriano N, Wright CR, Ashburner M: The Dichaete gene of Drosophila melanogaster encodes a SOX-domain protein required for embryonic segmentation. Development 1996, 122:3669-3676.

6. Guth SIE, Wegner M: Having it both ways: Sox protein function between conservation and innovation. Cell Mol Life Sci 2008, 65:3000-3018.

7. Phochanukul N, Russell S: No backbone but lots of Sox: Invertebrate Sox genes. Int J Bioc Cell Bio 2010, 42:453-464

8. Wegner M: SOX after SOX: SOXession regulates neurogenesis. Genes Dev 2011, 25:2423-2428

9. Buescher M, Hing FS, Chia W: Formation of neuroblasts in the embryonic central nervous system of Drosophila melanogaster is controlled by SoxNeuro. Development 2002, :4193-4203.

10. Overton P, Meadows L, Urban J, Russell S: Evidence for differential and redundant function of the Sox genes Dichaete and SoxN during CNS development in Drosophila. Development 2002, 129:4219-4228.

11. Miyagi S, Kato H, Okuda A: Role of SoxB1 transcription factors in development. Cell Mol Life Sci 2009, 66:3675-3684.
12. Cremazy F, Berta P, Girard F: Genome-wide analysis of Sox genes in Drosophila melanogaster. Mech Dev 2001, 109:371-375.

13. McKimmmie C, Woerfel G, Russell S: Conserved genomic organisation of group B Sox genes in insects. BMC Genet 2005, 6:26.21-26.15.

14. Wilson MJ, Dearden PK: Evolution of the insect Sox genes. BMC Evol Biol 2008, 8:120.

15. Sanchez-Soriano N, Russell S: The Drosophila Sox-domain protein Dichaete is required for the development of the central nervous system midline. Development 1998, 125:3989-3996.

16. Zhao G, Boekhoff-Falk G, Wilson BA, Skeath JB: Linking pattern formation to cell-type specification: Dichaete and Ind directly repress achaete gene expression in the Drosophila CNS. Proc Nat Acad Sci USA 2007, 106:3847-3852.

17. Zhao G, Skeath J: The Sox-domain containing gene Dichaete/fish-hook acts in concert with vnd and ind to regulate cell fate in the Drosophila neuroectoderm. Development 2002, 129:1165-1174.

18. Sanchez-Soriano N, Russell S: Regulatory mutations of the Drosophila Sox gene Dichaete reveal new functions in embryonic brain and hindgut development. Dev Biol 2000, 129:1165-1174.

19. Ma Y, Tel KC, Gao Y, Niemitz E, Mosher J, Mukherjee A, Mutsuddi M, Huseinovic N, Crews ST, Johnson WA, et al: Functional interactions between Drosophila bHLH/PAS, Sox, and POU transcription factors regulate CNS midline expression of the slit gene. J Neurosci 2000, 20:4596-4605.

20. Ambrosetti DC, Basilico C, Dailey L: Synergistic activation of the fibroblast growth factor 4 enhancer by Sox 2 and Oct-3 depends on protein-protein interactions facilitated by a specific spatial arrangement of factor binding sites. Mol Cell Biol 1997, 17:6321-6329.

21. Remenyi A, Lins K, Nissen $L$, Reinbold R, Scholer HR, Wilmanns M: Crystal structure of a POU/HMG/DNA ternary complex suggests differential assembly of Oct4 and Sox2 on two enhancers. Genes Dev 2003, 17:2048-2059.

22. Maurange C, Cheng L, Gould AP: Temporal Transcription Factors and Their Targets Schedule the End of Neural Proliferation in Drosophila. Cell 2008, 133:891-902

23. MacArthur S, Li XY, Li J, Brown JB, Chu HC, Zeng L, Grondona BP, Hechmer A, Simirenko L, Keranen SV, et al: Developmental roles of 21 Drosophila transcription factors are determined by quantitative differences in binding to an overlapping set of thousands of genomic regions. Genome Biol 2009, 10:R80.

24. Negre N, Brown CD, Ma L, Bristow CA, Miller SW, Wagner U, Kheradpour P, Eaton ML, Loriaux $\mathrm{P}$, Sealfon $\mathrm{R}$, et al: A cis-regulatory map of the Drosophila genome. Nature 2011, 471:527-531.

25. Brand $\mathrm{AH}$, Perrimon N: Targeted gene expression as a means of altering cell fates and generating dominant phenotypes. Development 1993, 118:401-415.

26. Kishi M, Mizuseki K, Sasai N, Yamazaki H, Shiota K, Nakanishi S, Sasai Y: Requirement of Sox2-mediated signalling for differentiation of early Xenopus neuroectoderm. Development 2000, 127:791-800.

27. Graham V, Khudyakov J, Ellis P, Pevny L: SOX2 functions to maintain neural progenitor identity. Neuron 2003, 39:749-765

28. Okuda Y, Ogura E, Kondoh H, Kamachi Y: B1 SOX coordinate cell specification with patterning and morphogenesis in the early zebrafish embryo. PLOS Genet 2010, 6:e1000936.

29. John A, Smith ST, Jaynes JB: Inserting the Ftz homeodomain into Engrailed creates a dominant transcriptional repressor that specifically turns off Ftz target genes in vivo. Development 1995, 121:1801-1813.

30. Vickers ER, Sharrocks AD: The use of inducible engrailed fusion proteins to study the cellular functions of eukaryotic transcription factors. Methods 2002, 26:270-280

31. Kambadur R, Koizumi K, Stivers C, Nagle J, Poole SJ, Odenwald WF: Regulation of POU genes by castor and hunchback establishes layered compartments in the Drosophila CNS. Genes Dev 1998, 12:246-260.

32. Ruiz-Gomez M, Ghysen A: The expression and role of a proneural gene, achaete, in the development of the larval nervous system of Drosophila. EMBO J 1993, 12:1121-1130.

33. Tear G, Harris R, Sutaria S, Kilomanski K, Goodman CS, Seeger MA: Commissureless controls growth cone guidance across the cns midline in Drosophila and encodes a novel membrane protein. Neuron 1996, 16:501-514

34. Jarman $A P$, Brand $M$, Jan $L Y$, Jan $Y N$ : The regulation and function of the helix-loop-helix gene, asense, in Drosophila neural precursors. Development 1993, 119:19-29. 
35. Bergsland M, Ramskold D, Zaouter C, Klum S, Sandberg R, Muhr J: Sequentially acting Sox transcription factors in neural lineage development. Genes Dev 2011, 25:2453-2464.

36. Kearney JB, Wheeler SR, Estes P, Parente B, Crew ST: Gene expression profiling of the developing Drosophila CNS midline cells. Dev Biol 2004, 275:473-492.

37. Kumar S, Konikoff C, Van Emden B, Busick C, Davis KT, Ji S, Wu LW, Ramos H, Brody T, Panchanathan $S$, et al: FlyExpress: visual mining of spatiotemporal patterns for genes and publications in Drosophila embryogenesis. Bioinformatics 2011, 27:3319-3320.

38. Herrmann C, Van de Sande B, Potier D, Aerts S: I-cisTarget: an integrative genomics method for the prediction of regulatory features and cisregulatory modules. Nuc Acids Res 2012, 40:e114.

39. Ma Y, Niemitz EL, Nambu PA, Shan X, Sackerson C, Fujioka M, Goto T, Nambu JR: Gene regulatory functions of Drosophila Fish-hook, a high mobility group domain Sox protein. Mech Dev 1998, 73:169-182.

40. Campos-Ortega JA: Early neurogenesis in Drosophila melanogaster. In The Development of Drosophila melanogaster vol II. Edited by Bate M, MartinezArias A. Long Island NY: Cold Spring Harbor Publ; 1993:1091-1130.

41. Szklarczyk D, Franceschini A, Kuhn M, Simonovic M, Roth A, Minguez P, Doerks T, Stark M, Muller J, Bork P, et al: The STRING database in 2011: functional interaction networks of proteins, globally integrated and scored. Nuc Acids Res 2011, 39:D561-D568.

42. Keleman K, Riberio C, Dickson BJ: Comm function in commissural axon guidance: cell-autonomous sorting of Robo in vivo. Nat Neurosci 2005, 8:156-163.

43. Wheeler JC, Vanderzwan C, Xu X, Swantek D, Tracey WD, Gergen JP: Distinct in vivo requirements for establishment versus maintenance of transcriptional repression. Nat Genet 2002, 32:206-210.

44. Brand M, Jarman AP, Jan LY, Jan YN: Asense is a Drosophila neural precursor gene and is capable of initiating sense organ formation. Development 1993, 119:1-17.

45. Thomas JB, Crews ST, Goodman CS: Molecular genetics of the singleminded locus: a gene involved in the development of the Drosophila nervous system. Cell 1988, 52:133-141.

46. de Celis JF, Llimargas M, Casanova J: Ventral veinless, the gene encoding the Cf1a transcription factor, links positional information and cell differentiation during embryonic and imaginal development in Drosophila melanogaster. Development 1995, 121:3405-3416.

47. Zhao G, Wheeler SR, Skeath JB: Genetic control of dorsoventral patterning and neuroblast specification in the Drosophila Central Nervous System. Int J Dev Biol 2007, 51:107-115.

48. Pearson BJ, Doe CQ: Specification of temporal identity in the developing nervous system. Annu Rev Cell Dev Biol 2004, 20:619-647.

49. Kan L, Isasena N, Zhang Z, Hu M, Zhao L-R, Jalali A, Sahni V, Kessler J: Sox1 acts through multiple independent pathways to promote neurogenesis. Dev Biol 2004, 269:580-594.

50. Chao AT, Jones WM, Bejsovec A: The HMG-box transcription factor SoxNeuro acts with Tcf to control Wg/Wnt signaling activity. Development 2007, 134:989-997.

51. Overton PM, Chia W, Buescher M: The Drosophila HMG-domain proteins SoxNeuro and Dichaete direct trichome formation via the activation of shavenbaby and the restriction of Wingless pathway activity. Development 2007, 134:2807-2813.

52. Bogdan S, Klambt C: Epidermal growth factor receptor signaling. Curr Biol 2001, 11:R292-R295.

53. Bernard P, Harley VR: Acquisition of SOX transcription factor specificity through protein-protein interaction, modulation of Wnt signalling and post-translational modification. Int J Biochem Cell Biol 2010, 42:400-410.

54. Karess RE: $\mathbf{P}$ element mediated germ line transformation of Drosophila. In DNA cloning Volume II. Edited by Glover DM. Oxford: IRL Press; 1985:121-142.

55. Patel NH: Imaging neuronal subsets and other cell types in whole mount Drosophila embryos and and larvae using antibody probes. In Drosophila melanogaster: Practical Uses in Cell Biology. vol. 44th edition. Edited by Goldstein LSB, Fyrbeg E. New York: Academic; 1994:445-456.

56. Birch-Machin I, Gao S, Huen D, McGirr R, White RA, Russell S: Genomic analysis of heat-shock factor targets in Drosophila. Genome Biol 2005, 6:R63.
57. Contrino S, Smith RN, Butano D, Carr A, Hu F, Lyne R, Rutherford K, Kalderimis A, Sullivan J, Carbon S, et al: ModMine: flexible access to modENCODE data. Nuc Acids Res 2012, 40:D1082-D1088

58. Nicol JW, Helt GA, Blanchard SG Jr, Raja A, Loraine AE: The Integrated Genome Browser: free software for distribution and exploration of genome-scale datasets. Bioinformatics 2009, 25:2730-2731.

59. Lyne R, Smith R, Rutherford K, Wakeling M, Varley A, Guillier F, Janssens H, Ji W, McLaren P, North P, et al: FlyMine: an integrated database for Drosophila and Anopheles genomics. Genome Biol 2007, 8:R129.

60. Buhler J, Ideker T, Haynor D: Dapple: improved techniques for finding spots on DNA microarrays. UW CSE Technical Report, UWTR 2000-08-05; 2000.

61. Huber W, Heydebreck AV, Sultmann H, Poustka A, Vingron M: Varience stabilization applied to microarray data calibration and to the quantification of differential expression. Bioinformatics 2002, 18:596.

62. Baldi P, Long AD: A Bayesian framework for the analysis of microarray expression data: regularized t -test and statistical inferences of gene changes. Bioinformatics 2001, 17:509-519.

doi:10.1186/1471-213X-13-1

Cite this article as: Shen et al:: Identifying targets of the Sox domain protein Dichaete in the Drosophila CNS via targeted expression of dominant negative proteins. BMC Developmental Biology 2013 13:1.

\section{Submit your next manuscript to BioMed Central and take full advantage of:}

- Convenient online submission

- Thorough peer review

- No space constraints or color figure charges

- Immediate publication on acceptance

- Inclusion in PubMed, CAS, Scopus and Google Scholar

- Research which is freely available for redistribution

Submit your manuscript at www.biomedcentral.com/submit
C) BioMed Central 